\title{
Receding-Horizon Multi-Step Optimization to Correct Non Viable or Unstable Transmission Voltages
}

\author{
Mevludin Glavic, Senior Member, IEEE, Mahdi Hajian, Student Member, IEEE, \\ William Rosehart, Senior Member, IEEE and Thierry Van Cutsem, Fellow, IEEE
}

\begin{abstract}
In this paper, a receding-horizon multi-step optimization is proposed to correct non viable transmission voltages and prevent long-term voltage instability. The proposed control scheme is based on real-time control, inspired by model predictive control, and steady state power-flow-based equations. In order to anticipate load behavior and avoid using dynamic equations in the control scheme, explicit formulations are used to model evolution of load with time. The simulation results of the proposed technique are presented on the Nordic32 test system.
\end{abstract}

Index Terms-real-time voltage control, long-term voltage instability, receding horizon, multi-step optimization, load shedding, capacitor switching compensation, generator voltage control.

\section{INTRODUCTION}

$\mathbf{V}$ OLTAGE control is an important aspect of system operation aimed at maintaining grid voltages within prescribed limits while accounting for generator reactive power reserves. Under certain operating conditions, systems can exhibit abnormal but stable voltage profiles that must be corrected. Furthermore, several power system blackouts around the world [1], [2] have stressed the importance of emergency voltage control to prevent voltage instability problems. To relieve operators from this delicate task, there is a need for automatic control schemes able to correct non viable or unstable voltages characterized by dramatic drops in bus voltages potentially leading to system collapse [3], [4].

The literature that deals with corrective control of voltages is often based on real-time tools[5]. In [6], a real-time voltage control scheme is considered to mitigate voltage violations and minimize transmission losses. One of the first automatic voltage control relying on a (sensitivity) model of the power system is the so-called coordinated secondary voltage control, which is in operation in two regional control centers of the French system [7]. Reference [8] discusses the possibility to use the algorithm of the French secondary voltage control to deal with emergency actions, such as load shedding.

One class of real-time approaches is based on Model Predictive Control (MPC), which consists of determining a

M. Glavic (glavic@montefiore.ulg.ac.be) is visiting researcher at the Department of Electrical Engineering and Computer Science (Montefiore Institute), University of Liège, Sart Tilman B37, B-4000 Liège, Belgium.

M. Hajian and W. Rosehart (mhajian, rosehart@ieee.org) are with the Department of Electrical and Computer Engineering, Schulich School of Engineering, University of Calgary, 2500 University Dr., Calgary, AB, Canada. T. Van Cutsem (t.vancutsem@ulg.ac.be) is with the Fund for Scientific Research - FNRS at the Department of Electrical Engineering and Computer Science of the University of Liège, Belgium. sequence of future control actions, based on measurements received and a model of the system evolution, applying the first step of the so computed sequence, and repeating the whole procedure at the next time step, when new measurements are collected[9], [10], [11], [12], [13], [14], [15], [16]. In order to capture the dynamic response of system, including load behavior, differential-algebraic equations have been often used inside of the controller optimization routine. A flexible secondary voltage control is proposed in [9] based on MPC in which both static and dynamic optimization subproblems are used. In [10], emergency voltage control is addressed using MPC based on sensitivity analysis calculated via system dynamic equations. In [11], the coordination of generator voltages, tap changers, and load shedding is studied using tree search optimization techniques. In [12], a coordinated voltage control framework is developed based on non-linear system equations using Euler state prediction and pseudo gradient evolutionary programming. In [13], MPC of load is used to determine minimum amount of load shedding to restore system voltages. A centralized quadratic programming MPC formulation is considered in [14] to optimally coordinate generator voltage references and load shedding and solved via Lagrangian decomposition. In [15], a control switching strategy of shunt capacitors is presented by means of MPC to prevent voltage collapse and maintain a desired stability margin after a contingency.

In this paper, a Receding-Horizon Multi-Step Optimization (RHMSO) based real-time control approach is proposed to correct non viable transmission voltages and mitigate longterm voltage instability. The controls considered in this paper are shunt capacitors, generator voltage references, and load shedding. The proposed RHMSO controller is inspired by MPC formulation in the sense of implementing multi-step control actions and modifying the controls based on the feedback received from the system. However, it differs from standard MPC formulations by the way the future system evolution is modeled. Traditionally, MPC schemes embed a state transition equation of the type $\mathbf{x}^{k+1}=\mathbf{f}\left(\mathbf{x}^{k}, \mathbf{u}^{k}\right)$ (where $\mathbf{x}$ is the state and $\mathbf{u}$ is the control vector) sometimes obtained by algebraization of differential equations and often linearized around successive operating points. The proposed RHMSO scheme uses an explicit model of load power evolution with time, thereby avoiding to rely on detailed load models which are very often uncertain in practice. By so doing, the model is of similar complexity compared to standard optimal power 
flows relying on power flow equations. An additional contribution of this paper is to demonstrate that accounting for the load power restoration effect produced by Load Tap Changers (LTCs) after a disturbance can lead to satisfactory voltage control. Finally, another distinctive feature of the proposed scheme is the handling of generator reactive power limits, which takes advantage of their overexcitation capability. The proposed RHMSO scheme is designed to both mitigate voltage instability and to efficiently correct non viable but stable voltage profiles.

The paper is organized as follows. Basic assumptions regarding general control strategy are presented in Section II. The description of a single-step optimization approach to control transmission voltages is provided in Section III. The RHMSO scheme is developed in Section IV, with simulation results presented in Section V. Section VI lists conclusions.

\section{BASIC ASSUMPTIONS}

We focus on the control of transmission voltages over a period of several minutes after a disturbance. In this time interval, the system typically evolves under the long-term dynamics of LTCs controlling distribution voltages and OverExcitation Limiters (OELs) protecting synchronous generators. Other load power restoration mechanisms may also take place, as well as some slow controls such as automatic shunt device switching. The short-term dynamics are assumed stable since the proposed controller focuses on long-term dynamics [4].

We assume that system measurements are collected and processed by a state estimator in the region of interest, so that "snapshots" of voltage measurements and bus power injections are available at a sampling rate in the order of - say - $10 \mathrm{~s}$. To this purpose, standard SCADA (Supervisory Control And Data Acquisition) measurements can be advantageously enriched with synchronized phasor measurements provided by PMUs (Phasor Measurements Units).

In practice transmission voltages are requested to lie within a specified range of values:

$$
\mathbf{V}^{\min } \leq \mathbf{V} \leq \mathbf{V}^{\max }
$$

where $\mathbf{V}$ denotes the vector of voltage magnitudes at the $N$ buses, and $\mathbf{V}^{\min }$ and $\mathbf{V}^{\max }$ are the corresponding admissible limits. The problem of concern here is to correct voltages that leave the specified range of values. Optimal adjustments of voltages within those limits, for instance to minimize transmission losses, is outside the scope of this paper.

To correct unacceptable voltages, the following controls are assumed to be available (listed in decreasing order of preference): shunt compensation, generator voltages and load curtailment. Shunt compensation is given higher preference since the unbundling of transmission and generation activities could make the control of generator voltages by TSOs more difficult or more expensive than in the vertically integrated industry. The formulation easily accommodates additional controls such as generator active powers and ratios of transformers located in the transmission system (LTCs controlling distribution voltages are assumed to act automatically and are considered embedded in loads).
Any correction of transmission voltages should obey the limits on the reactive power produced by generators:

$$
\mathbf{Q}^{\min } \leq \mathbf{Q} \leq \mathbf{Q}^{\max }
$$

where $\mathbf{Q}$ denotes the vector of reactive power productions, and $\mathbf{Q}^{\min }$ and $\mathbf{Q}^{\max }$ are the corresponding lower and upper limits. Each limit should be updated with the corresponding active power production and terminal voltage, in accordance with the generator capability curves. To this purpose, the optimization problems presented in this paper utilize equation (3.51) from [4].

Let us consider a large disturbance, such as generator or line tripping, occurring at $t=0$. Let us denote by $\mathbf{s}$ the vector of load active and reactive powers, and let $\mathbf{s}\left(0^{+}\right)$be its value shortly after the disturbance (i.e. after short-term dynamics have died out). Most loads have their active and reactive powers decreasing with distribution voltages. Hence, the initial impact of the disturbance will be a decrease in the components of $\mathbf{s}$ in areas importing power, while an increase can be experienced on loads located at the sending end of a transmission corridor, after the outage of a transmission link within that corridor.

An important component of the long-term dynamics is the load power restoration through the restoration of distribution voltages by automatic LTCs. Under its effect, one can assume that the $\mathbf{s}$ vector will progressively recover its pre-disturbance value, denoted $\mathbf{s}\left(0^{-}\right)$. At load buses where curtailment has been applied, LTC action will result in recovery of the not curtailed portion of the load. In practice, LTC deadbands may lead to somewhat different distribution voltages, and hence powers of the voltage sensitive loads.

\section{THE SINGLE-STEP OPTIMIZATION APPROACH}

The correction of unsatisfactory voltages can be formulated as the problem of restoring feasibility of the operating point, at minimum cost, and anticipating load power restoration. The corresponding Optimal Power Flow (OPF) problem, referred to in the sequel as Single-Step Optimization(SSO), can be written as:

$$
\min _{\mathbf{u}, \mathbf{x}} \sum_{i=1}^{n} c_{i}\left[u_{i}-u_{i}^{o}\left(0^{-}\right)\right]^{2}
$$

subject to : $\mathbf{g}\left(\mathbf{x}, \mathbf{u}, \mathbf{s}\left(0^{-}\right)\right)=\mathbf{0}$

$$
\begin{aligned}
& \mathbf{u}^{\text {min }} \leq \mathbf{u} \leq \mathbf{u}^{\text {max }} \\
& \mathbf{V}^{\text {min }} \leq \mathbf{V}(\mathbf{x}, \mathbf{u}) \leq \mathbf{V}^{\text {max }} \\
& \mathbf{Q}^{\text {min }} \leq \mathbf{Q}(\mathbf{x}, \mathbf{u}) \leq \mathbf{Q}^{\text {max }}
\end{aligned}
$$

In these relations, $\mathbf{x}$ is the state vector, which includes load bus voltage magnitudes as well as other variables such as the bus voltage phase angles. $\mathbf{u}$ is the vector of $n$ controlled variables, including shunt susceptances, generator voltage setpoints and load demands. $\mathbf{u}\left(0^{-}\right)$denotes the pre-disturbance value of $\mathbf{u}$. Elements of the bus voltage magnitude vector $\mathbf{V}$ are either elements of $\mathbf{x}$, if they are not directly controlled, such as a load bus, or elements of $\mathbf{u}$ if they are directly controlled, such as a generator bus. 
The objective (3a) minimizes the deviations of control variables. The weights $c_{i}$ reflect the respective costs of the various controls, as mentioned in Section II. Thus, a larger value is appropriate for generator voltage setpoints than for shunt susceptances, while a much larger value should be considered for load shedding in order to resort to the latter only when the other means are insufficient. A Euclidean norm is utilized in the objective to push the solution towards using more than one control action, which tends to increase reliability if there is a control signal failure.

The steady-state operation of the system in the postdisturbance post-control configuration is modeled using (3b). In the simplest case, the network active and reactive power flow equations are used, although one can resort to a more accurate Quasi Steady-State (QSS) model, as detailed in [17]. Note that load power restoration is anticipated by setting the load powers to $\mathbf{s}\left(0^{-}\right)$in these equations. To preserve simplicity, the active power productions of generators are not shown explicitly in (3b). If standard power flow equations are used, a distributed slack bus formulation is appropriate to share the active power adjustments over all generators participating in frequency control. The constraints $(3 c)$ deal with limits on control variables, the bounds being possibly a function of $\mathbf{u}\left(0^{-}\right)$. The constraints (3d) and (3e) have been already commented.

In the context of real-time control, the above SSO scheme suffers from major deficiencies:

1) any model inaccuracy will be reflected on the final settings and operating point; such an open-loop scheme offers no chance to correct the consequences of modeling errors. A similar remark applies to measurement noise;

2) no chance is given to compensate for control changes that are not implemented as expected due to failures or uncertainty in system behavior;

3) it does not (easily) allow accounting for new events taking place in the system;

4) it provides a "target state" but not the transitions to reach that state. In standard OPF implementations, this task is left to the operator. The latter should be relieved from the burden of implementing the computed control changes. This was one of the main motivations for implementing secondary voltage control in some countries [7]. It becomes even more important in the stressed voltage instability situations considered in this paper. If large voltage corrections have to be applied to generators, implementing them in full and "one at a time" may lead to unacceptable transient variations of the generator reactive power outputs;

5) As another consequence of not considering the transitions to the target stage, advantage cannot be taken of the temporary field current overload capability of generators. The latter contributes to supporting network voltages.

As discussed in the Introduction, a scheme inspired of MPC can deal with the above issues.

\section{THE MULTI-STEP OPTIMIZATION APPROACH}

\section{A. The Optimization Problem}

An RHMSO approach involves the following [16]:

1) at a given time step $k$, collect measurements

2) compute the sequence of controls $\left(\mathbf{u}^{k+1}, \mathbf{u}^{k+2}, \ldots, \mathbf{u}^{k+K}\right)$ that should be applied at the $K$ future time steps to bring all voltages and powers in the desired intervals after $K^{\prime}$ steps

3 ) at step $k+1$, apply the first element $\mathbf{u}^{k+1}$ of the socomputed sequence

4) $k:=k+1$; repeat steps 1 to 3 .

$K$ is referred to as the control horizon and $K^{\prime}$ as the prediction horizon. It is recommended to have $K^{\prime} \geq K$ [16]. With the simplified dynamics considered here, there is no clear advantage in choosing $K^{\prime}>K$, and we thus take $K^{\prime}=K$.

We propose the following multi-step optimization problem to be solved at step 2 of the above procedure:

$$
\begin{gathered}
\mathbf{m i n}^{k+1}, \ldots, \mathbf{u}^{k+K} \sum_{j=k+1}^{k+K} \sum_{i=1}^{n} c_{i}\left(u_{i}^{j}-u_{i}^{j-1}\right)^{2} \\
\mathbf{x}^{k+1}, \ldots, \mathbf{x}^{k+K} \\
\text { s.t. } \quad \mathbf{g}\left(\mathbf{x}^{j}, \mathbf{u}^{j}, \mathbf{s}^{j}\right)=\mathbf{0} \quad j=k+1, \ldots, k+K \\
\mathbf{u}^{\text {min }} \leq \mathbf{u}^{j} \leq \mathbf{u}^{\text {max }} \quad j=k+1, \ldots, k+K \\
\left|\mathbf{u}^{j}-\mathbf{u}^{j-1}\right| \leq \mathbf{\Delta} \quad j=k+1, \ldots, k+K \\
\mathbf{V}^{\text {min }} \leq \mathbf{V}^{k+K}(\mathbf{x}, \mathbf{u}) \leq \mathbf{V}^{\text {max }} \\
\mathbf{Q}^{\text {min }} \leq \mathbf{Q}\left(\mathbf{x}^{k+K}, \mathbf{u}^{k+K}\right) \leq \mathbf{Q}^{\text {max }} \\
\mathbf{Q}_{i}^{\text {min }} \leq \mathbf{Q}\left(\mathbf{x}^{j}, \mathbf{u}^{j}\right) \leq \mathbf{Q}_{i}^{\text {max }} \quad i \in \mathcal{I}(k) ; \\
j=k+1, \ldots, k+K-1
\end{gathered}
$$

with a notation similar to the one of Section III.

The objective (4a) is similar to (3a) but the control effort is now distributed over the next $K$ time steps. Equations (4b) involve one set of equations of the type (3b) per time step. The sequence of load power values $\left\{\mathbf{s}^{k+1}, \ldots, \mathbf{s}^{k+K}\right\}$ to be used in (4b) is discussed in Section IV-B. The constraints (4c) are obtained by repeating (3c) over the $K$ steps, while the constraints (4d) aim at limiting the rate of change of the controls, where vector $\boldsymbol{\Delta}$ models the rate of change limits of the various control actions, for instance generator ramping limits.

The inequalities (4e) impose to have, at the end of control horizon, all voltages back in the admissible intervals. Let us emphasize that only those final voltages are required to be within limits, not the intermediate values $\left\{\mathbf{V}^{k+1}, \ldots, \mathbf{V}^{k+K-1}\right\}$.

Similarly, the inequalities (4f) impose all generators to have their reactive power productions within limits at the end of control horizon. However, the constraints $(4 \mathrm{~g})$, relative to intermediate time steps, apply to only a subset $\mathcal{I}(k)$ of generators, determined as explained in Section IV-D. The choice of $\mathrm{K}$ will be discussed later on (see Section V-I).

\section{B. Load Power Restoration}

Many of the previous works on MPC applied to voltage control used generic models of load dynamics. Those models 


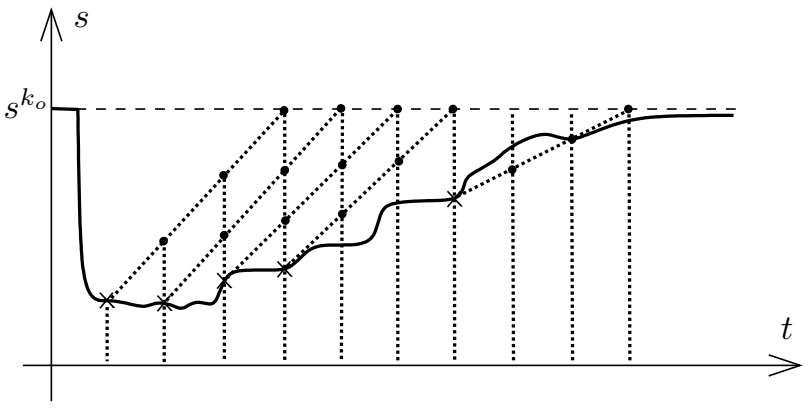

Fig. 1. Linear load power recovery assumed in the controller.

may fail capturing the whole complexity of load responses. For instance, the single time-constant recovery model [18], [19] may not reproduce the behavior of (one or several levels of) LTCs operating with various delays. However, there is quite some uncertainty on the parameters involved in load models, in particular the variation of powers with voltage. Hence, one may question the added value of embedding those models in a controller, at the cost of increasing its computational complexity. Instead, the controller should operate satisfactorily with minimal information about the load behavior.

One contribution of this paper is to demonstrate that a satisfactory voltage control indeed can be obtained without tracking the real (and in many cases not well known) load response, but just accounting for the load power restoration effect produced by LTCs after a disturbance. To this purpose, an explicit evolution of load powers with time is assumed, as detailed hereafter.

Let $k_{o}$ be the last time step where all bus voltages and all reactive generations were within their limits $(3 \mathrm{~d}, 3 \mathrm{e})$. The load powers collected at that time step, i.e. $\mathbf{s}^{k_{o}}$, are taken by the controller as reference until it succeeds bringing all voltages and reactive generations within limits. Let $\mathbf{s}^{k}$ be the vector of load powers collected at the $k$-th time step, where $k>k_{o}$. Assuming that load powers are going to recover to $\mathbf{s}^{k_{o}}$ in $K$ steps, the future values used in (4b) are:

$$
\mathbf{s}^{j}=\mathbf{s}^{k}+\alpha^{j}\left(\mathbf{s}^{k_{o}}-\mathbf{s}^{k}\right) \quad j=k+1, \ldots, k+K
$$

with $\alpha^{k+1}<\alpha^{k+2}<\ldots<\alpha^{k+K}=1$. The procedure is illustrated in Fig. 1, for a linear recovery, i.e. $\alpha^{j}=(j-k) / K$, over $K=3$ steps. The solid line is the time evolution of one component of $\mathbf{s}$ after a disturbance; the sampled values are shown with " $x$ ". The future values determined from (5) are shown with dots. Note that the formula is re-applied at each step, starting from the newly collected $\mathbf{s}^{k}$ value.

\section{Controller Activation}

As long as all bus voltages and generator reactive powers are within limits at the present $(j=k)$ and future $(j=k+$ $1, \ldots, k+K)$ time steps, no control changes will be issued, since the trivial solution of (4) is $\mathbf{u}^{j}=\mathbf{u}^{k}, j=k+1, \ldots, k+$ $K$. On the contrary, a sequence of controls will be issued in response to measured voltages or reactive powers leaving the specified intervals. Once the measured voltages and/or reactive powers have returned to their specified intervals, no control changes will be issued.

Furthermore, it may be appropriate to trigger the computation of a new control sequence upon detection of a topological change. Indeed, shortly after this change, it may happen that all measured voltages and reactive powers still lie within their limits while their future values (taking load power restoration into account) do not. This infeasibility of future states will trigger control changes, allowing the controller to anticipate the effective violation of the limits.

\section{Handling of Generator Reactive Power Limits}

It is well known that reactive power limitations of generators may lead to non viable or even unstable voltages.

A synchronous generator may temporarily operate with its field current above the permanent admissible value, until the OEL acts to reduce the latter. It is important to model and utilize this overexcitation capability since it contributes to keeping voltages under AVR control.

In the model of system evolution, one could think of anticipating the moment where OELs will come into play. In practice, however, neither the tuning of this protection nor the exact evolution of the field current are likely to be known from the control center hosting the RHMSO controller.

This led us to consider the successive OEL activations as additional disturbances applied to the system. The multi-step control strategy handles generator limits as follows:

1) if a generator operates above its capability, the corresponding reactive power limit is not enforced at the intermediate steps but only at the end of control horizon, to take benefit of its overexcitation while anticipating the OEL effect. Thus, the generator of concern is involved in $(4 \mathrm{f})$ but not in $(4 \mathrm{~g})$;

2) if the generator operates below its limit, the latter is specified as a constraint at all future steps, to avoid further degradation of the operating conditions. Thus, that generator is involved in both (4f) and (4g);

3) once a generator has its field current limited by its OEL, the constraint $(4 \mathrm{~g})$ becomes satisfied and is included at all future time steps.

\section{E. Handling of Generator Voltage Setpoints}

Due to the presence of steady-state errors in the Automatic Voltage Regulators (AVRs), many generating units have terminal voltages somewhat different from their AVR setpoints [4]. To deal with this discrepancy, one option is to resort to a QSS model of the generator, accounting for the finite open-loop AVR gain. If instead a standard network power flow model is used, as in the tests reported in this paper, only the generator terminal voltages are involved in the optimization problem (4). However, experience shows that a change in the AVR setpoint results in an almost equal change of terminal voltage. Hence, a change $V_{i}^{k+1}-V_{i}^{k}$ of the $i$-th generator terminal voltage can be implemented as an equal change of the AVR setpoint of that generator. By so doing, the RHMSO controller provides setpoint corrections instead of setpoint values. 
No reference is made in this paper to static var compensators, but the latter can be handled in the same way as generators, with obviously no overload capability.

\section{F. Relationship with Model Predictive Control}

The proposed scheme bears the spirit of MPC and inherits its already mentioned advantages. However, MPC may bring its own problems. In particular, if the uncontrolled system is unstable, care has to be taken so that the controller stabilizes the system. This problem has been tackled in the MPC literature and practice [20], [21]. Among the proposed solutions, the terminal constraint-set method recommended in e.g. [16], [21] consists of imposing the terminal state vector to lie in a set defined by inequality constraints. The inequalities $(4 \mathrm{e}, 4 \mathrm{f})$ are constraints of this type. However, they do not involve the whole state vector. In spite of this restriction, the approach has been found to work properly, provided the final states $\mathbf{V}^{k+K}$ and $\mathbf{Q}\left(\mathbf{x}^{k+K}, \mathbf{u}^{k+K}\right)$ involved in $(4 \mathrm{e}, 4 \mathrm{f})$ correspond to operating points with load powers restored as described in Section IV-B. This important aspect is illustrated in Section V-C.

\section{Simulation RESUlts}

\section{A. Test System, Models and Tools}

The proposed controller is demonstrated on a variant of the Nordic32 test system [22] previously used in [23]. The oneline diagram is shown in Fig. 2. It includes 52 buses and 20 synchronous machines (denoted with a g, followed by the machine number). The long-term system evolution is driven by LTCs and OELs, acting after various delays.

A detailed dynamic model (under the classical phasor approximation) of generators, AVRs and speed governors was considered to validate the controller in realistic conditions, with measurements affected by transients. The loads connected to the LTC-controlled distribution buses behave as constant current (resp. impedance) for the active (resp. reactive) power. This is not known by the RHMSO controller, which processes (in the $\mathbf{s}$ vectors) the active and reactive powers entering the distribution transformers on the transmission side.

This model was simulated with the MATLAB/SIMULINKbased tool described in [24]. The optimization problem (4) was solved in the GAMS-IDE environment [25], interfaced with SIMULINK through the MATGAMS interface documented in [26]. In GAMS, the best performances were achieved with IPOPT, a primal-dual interior-point nonlinear solver (with filter line-search method) [27], [28]. The simulations were completed using a Windows machine with Intel Core Duo $1.50 \mathrm{GHz} \mathrm{CPU}$ and $2 \mathrm{~GB}$ of RAM.

\section{B. RHMSO Controller Settings}

The proposed RHMSO controller can adjust: the 20 generator voltages in the range $\left[\begin{array}{ll}0.95 & 1.10\end{array}\right] \mathrm{pu}$, shunt compensation by steps of 10 Mvar at buses 1022, 1043, and 1044 and up to $30 \%$ of the loads at buses 1022, 1041, 1042, 1043, 1044, 1045, and 2031. The relative costs assigned to these controls are: $10^{0}$ for load shedding, $10^{-3}$ for generator voltages and

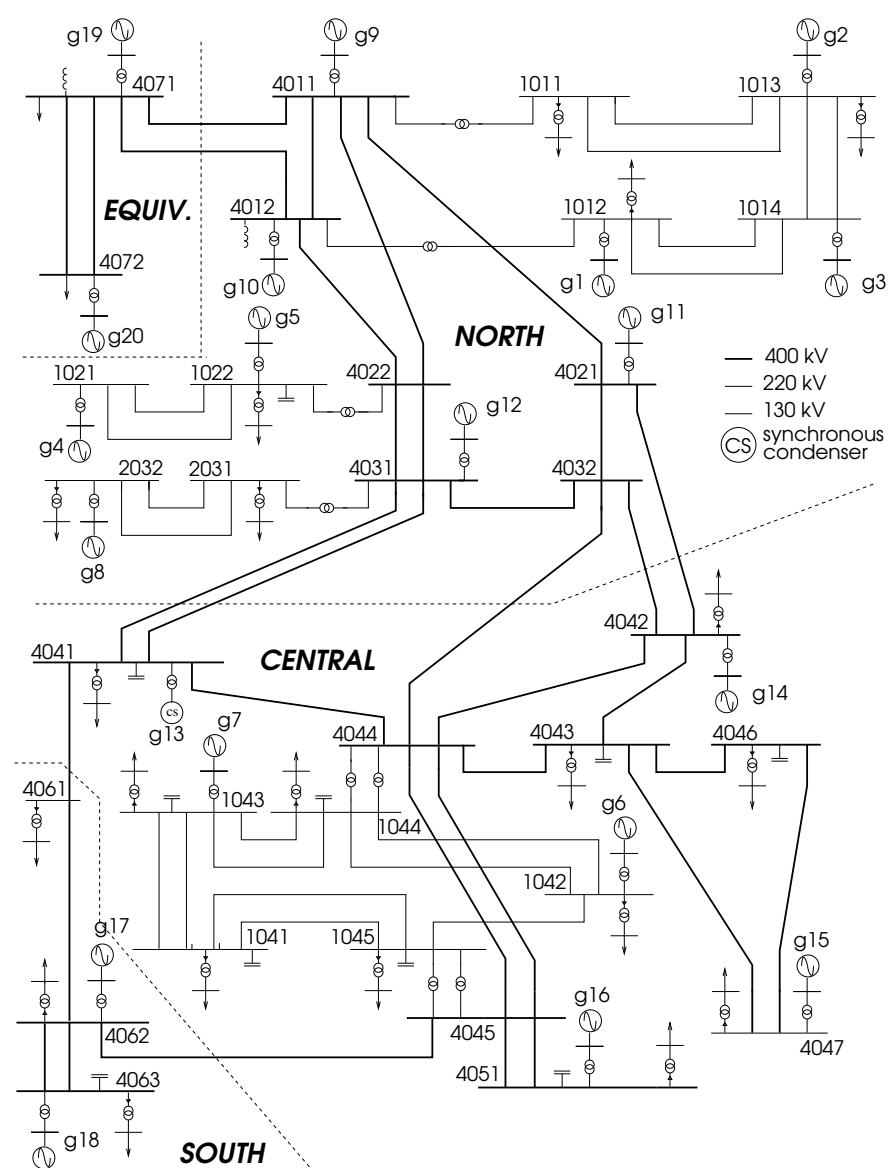

Fig. 2. One-line diagram of Nordic32 test system.

$10^{-6}$ for shunt compensation. Although shedding is heavily penalized, the optimization (4) might result in a small load curtailment while the other controls would suffice. To avoid this, any load shedding smaller than 0.1 MW is ignored.

For simplicity, shunt compensation has been treated as a continuous variable rounded off to the nearest multiple of 10 Mvar. The multi-step optimization easily accommodates such deviations with respect to the theoretical optimum.

A maximum rate of change of controls $\Delta$ (see Eq. 4d) is specified on generator voltages only, and is set to 0.05 pu. There is no limit imposed on the variation of capacitor susceptances or load powers.

The sampling period is 10 seconds. The control (and prediction) horizon has been set to $K=3$, which corresponds to $30 \mathrm{~s}$ after the measurements have been received.

\section{Case 1: Stabilization of a Voltage Unstable Scenario}

This case involves the outage of transmission line 40324044 (see Fig. 2) at $t=12 \mathrm{~s}$. The evolution of three transmission bus voltages is shown in Fig. 3. The voltages decline owing to LTCs attempting to restore distribution voltages, as well as field current limitations (on g14 at $t=99.2$, g12 at $t=103.4, \mathrm{~g} 6$ at $t=108.8, \mathrm{~g} 15$ at $t=116.8, \mathrm{~g} 7$ at $t=149.3$, and g16 at $t=152.3 \mathrm{~s}$ ) until collapse takes place at $158.9 \mathrm{~s}$, right after $\mathrm{g} 7$ and $\mathrm{g} 16$ become limited.

The solid, dashed and dash-dotted curves in Fig. 4 show the voltage evolution stabilized by the proposed RHMSO 


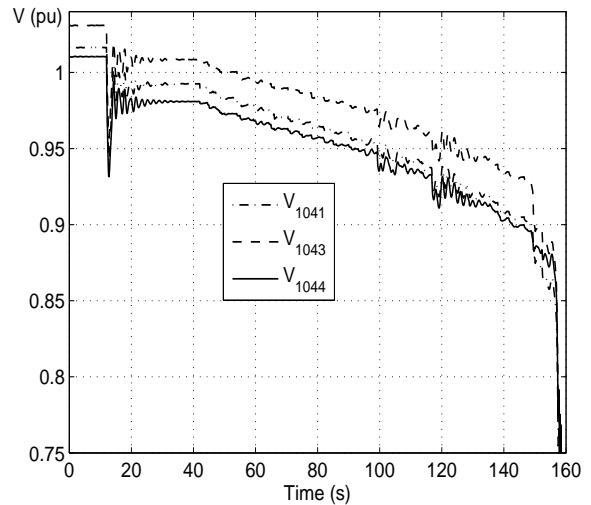

Fig. 3. Case 1: unstable system response.

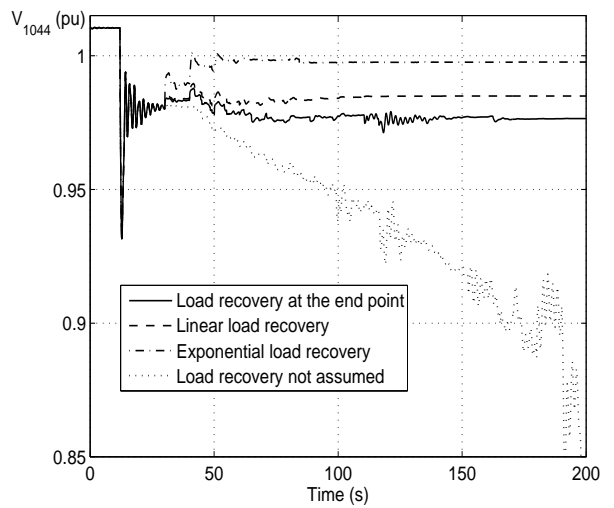

Fig. 4. Case 1: voltage at bus 1044 with the proposed controller; various load recovery assumptions.

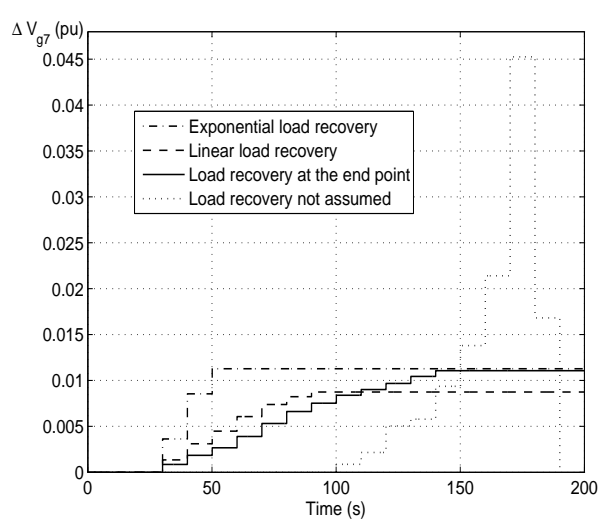

Fig. 5. Case 1: voltage of generator G7 modified by the proposed controller. controller. The latter switches on capacitors very early (in between $t=30$ and $t=50 \mathrm{~s}$ ) and modifies generator voltage setpoints during some $120 \mathrm{~s}$, but does not shed any load. An example of generator voltage variation is provided in Fig. 5.

The three curves were obtained assuming that load power recovers respectively:

- linearly as in (5), i.e. $\alpha^{k+1}=\frac{1}{3}, \alpha^{k+2}=\frac{2}{3}, \alpha^{k+3}=1$

- exponentially, i.e. $\alpha^{k+1}=0.6, \alpha^{k+2}=0.9, \alpha^{k+3}=1$

- at the end point only: $\alpha^{k+1}=\alpha^{k+2}=0, \alpha^{k+3}=1$.

Figure 5 shows that the linear recovery offers a compromise between the "aggressive" control with small settling time of exponential recovery and the smooth control with longer settling time of end-point recovery. The latter demonstrates that even an unrealistic load power recovery yields an acceptable controller response. What matters is to consider that load power will eventually restore. To confirm this, the same figure shows, with the dotted lines, the system evolution with no load recovery, i.e. the measured load powers are used in all eqs. (4b). The controller cannot stabilize the system, in spite of pronounced, but lately applied, control actions.

For the above reasons, linear recovery was adopted in all tests reported hereafter.

Figure 6 illustrates the ability to deal with generator reactive power limits. It shows the field current of g12, selected for being at some distance of buses 1043 and 1044 where shunt capacitors are switched (and obviously decrease the field current of nearby generators). The dashed line refers to the case without the RHMSO controller; the activation of the OEL at $t \simeq 103 \mathrm{~s}$ is easily identified. With the controller in operation (solid line), the field current is kept below its $1.9 \mathrm{pu}$ limit until $t=140 \mathrm{~s}$. From there on, the limit is slightly exceeded but for a short duration. Hence, the machine is kept under AVR control over the whole simulation. With the SSO approach it is not possible to take advantage of field current over-excitations since the control actions are computed with all limits enforced at once.

\section{Case 2. Same Unstable Case Stabilized by Load Shedding}

The same case is considered but with capacitor switching disabled and generator voltages prevented from increasing above their pre-disturbance values, in order to force the RHMSO controller to shed load. Voltages are indeed stabilized. For instance, the one at bus 1044 (considered in Fig. 4) settles to $0.978 \mathrm{pu}$ after some 100 seconds.

The total curtailed power is 106.7 MW. Shedding takes place very progressively over the seven (partly) interruptible loads as shown in Fig. 7. The exponential shape of each curve is noteworthy. The case shown involves some tiny load curtailments. However, tests were performed in which any load shedding smaller than some threshold was assumed impractical and was merely not implemented. In all cases, the RHMSO scheme was able to subsequently compensate for the not implemented actions with larger shedding steps, at the price of a somewhat more oscillatory response.

\section{E. Case 3. Correction of Low but Stable Voltages}

The same line outage is considered but with a lower initial load in the Central area (see Fig. 2), so that the system is longterm stable. However, some voltages fall below the $V^{\text {min }}=$ $0.95 \mathrm{pu}$ limit, as shown by the dashed curve in Fig. 8.

The same figure shows, with the solid line, the voltage with the proposed controller in operation. 40 Mvar of shunt capacitors are switched on as follows: 10 Mvar at bus 1043 at $t=30,60$ and $100 \mathrm{~s}, 10$ Mvar at bus 1044 at $t=30 \mathrm{~s}$. Neither generator voltages nor loads were modified in this scenario, which illustrates the controller ability to adjust to the severity of the situation.

\section{F. Case 4. Stabilization of a Load Increase Scenario}

Starting from the operating point of Cases 1 and 2, the loads at buses 1041, 1042, 1043, 1044 and 1045 were linearly increased with time, at a rate of $7.2 \mathrm{MW} / \mathrm{min}$ (in total) and until $t=530 \mathrm{~s}$. The loadability limit (significantly impacted by the generator excitation limitations) was crossed before reaching this time, and since LTCs attempt to restore load powers, longterm voltage instability followed. The resulting degradation eventually made g6 lose synchronism, at $t=518.9 \mathrm{~s}$, soon after its field current became limited.

In order to use all three types of controls, the available shunt compensation was limited to 10 Mvar at each of the 

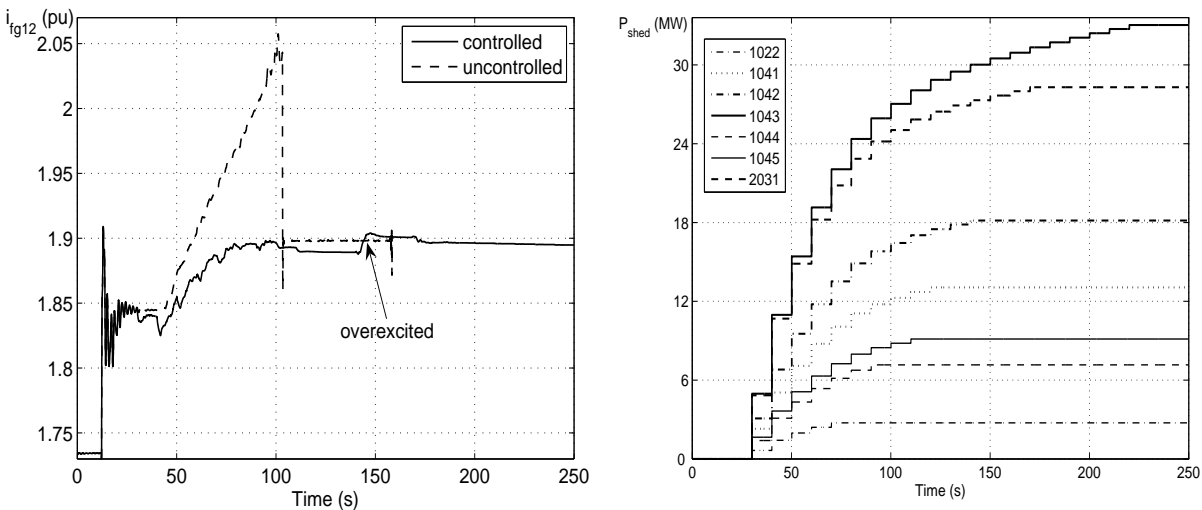

Fig. 6. Case 1: field current of g12 with and without Fig. 7. Case 2: cumulated load shedding. the proposed controller.

same three buses, while upper bounds on generator voltages were decreased to $0.01 \mathrm{pu}$ above the pre-disturbance values.

Figure 9 shows the system responses without and with the RHMSO controller. The latter smoothly stabilizes the system, which settles to a new long-term equilibrium at $t \simeq 650 \mathrm{~s}$.

The system operating state leaves the allowed intervals at $t=330 \mathrm{~s}$ for the first time. From there on, the load powers at the last feasible point are used as final values in the linear load recovery, except if the newly measured load power has a larger value, in which case this measurement is used at all $K$ future steps. This provides a simple way to track load increases. A similar procedure would apply to low load and high voltage conditions.

First, the controller resorts to shunt compensation, switching on the available $30 \mathrm{Mvar}$ at $t=340 \mathrm{~s}$. Then, it adjusts the generator voltages, as shown for three of them in Fig. 10. In the last resort, it curtails loads, as shown for three of them in Fig. 11. The total power shed is 77.0 MW.

\section{G. Case 5: Simulating Control Failures}

An RHMSO scheme is known for being able to adjust to changing conditions in the course of controlling the system, and hence inherently offers robustness with respect to both modeling uncertainties and component failures. The latter are considered here. We merely show how the controller faces a situation where actuators fail implementing the previously computed actions, and no information is provided about this failure. Techniques for enhancing fault-tolerance (e.g. fault detection and isolation [16]) are thus not considered.

Case 1 is repeated with the AVRs of generators g13, g14 and g16 as well as shunt capacitors at buses 1022 and 1044 not implementing the orders sent by the RHMSO controller. Figure 12 shows that the resulting voltage evolution only slightly departs from the one with all control changes applied correctly, in spite of the fact that the controller is not aware of the failures. Figure 13 shows how it compensates, after $t=70 \mathrm{~s}$, for the unsatisfactory system response, by acting more on the voltage of $\mathrm{g} 7$ (not subject to failure).

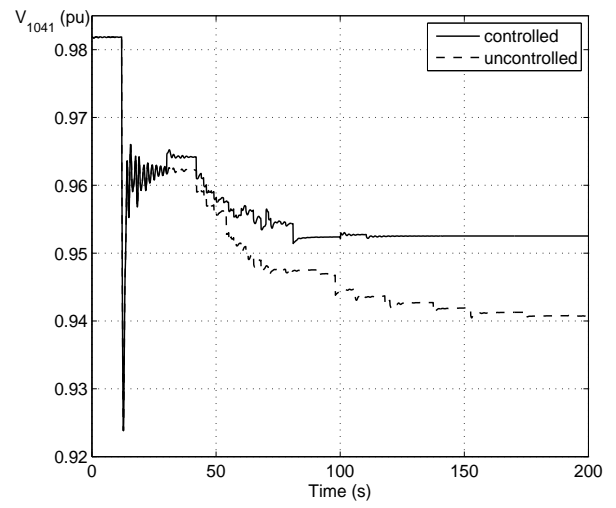

Fig. 8. Case 3: voltage at bus 1041 without and with the proposed controller.

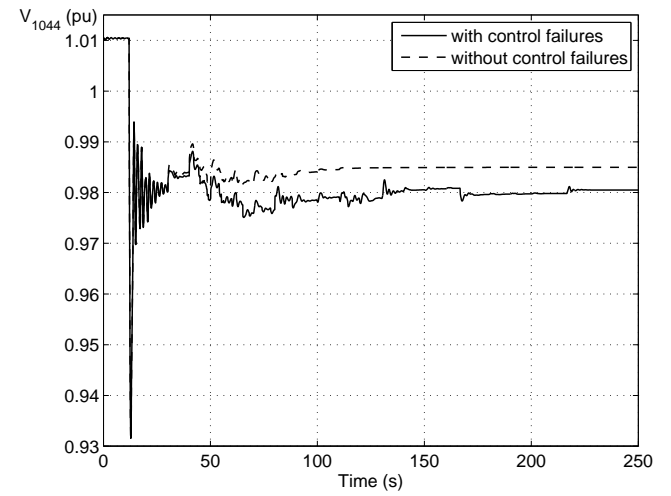

Fig. 12. Case 5: voltage response with and without component failure.

\section{H. Comparison with Single-Step Optimization}

In this section, the performance of the proposed multi-step approach is compared against a single-step approach (SSO considered in Section III) by simulating Case 2 presented in Section V-D. A comparison of computational burden for both approaches is included in Section V-J.

It was first assumed that an accurate system model is available to both approaches.

For the case scenario of concern, it was observed that the SSO approach is able to save the system, even with a bit less load shedding compared to the RHMSO approach (99.5 MW versus 106.7 MW). In fact, by varying generator parameters, such as decreasing the limits of generators g6 and g7, cases were found with the SSO approach shedding a little more load than the RHMSO approach. Thus, the total control effort is similar with both approaches. However, since load is curtailed by SSO at one time instant, more oscillations occur and consequently the settling time increases.

Next, control failures on variables were assumed to investigate performances in less optimal conditions.

In the first scenario considered, signals to switch the shunt capacitor at bus 1043 were blocked to simulate control failure of this component. The response obtained with the SSO approach, illustrated with the voltage magnitude at bus 1044 , is shown with dash-dotted line in Fig. 14. The SSO is not 


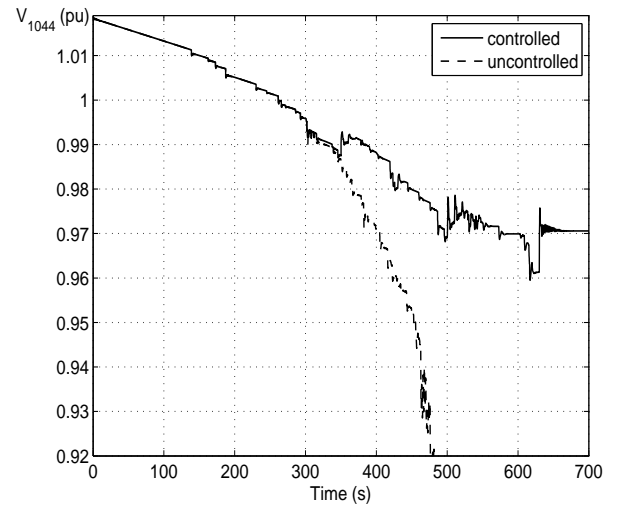

Fig. 9. Case 4: voltage at bus 1044 without and with the proposed controller.
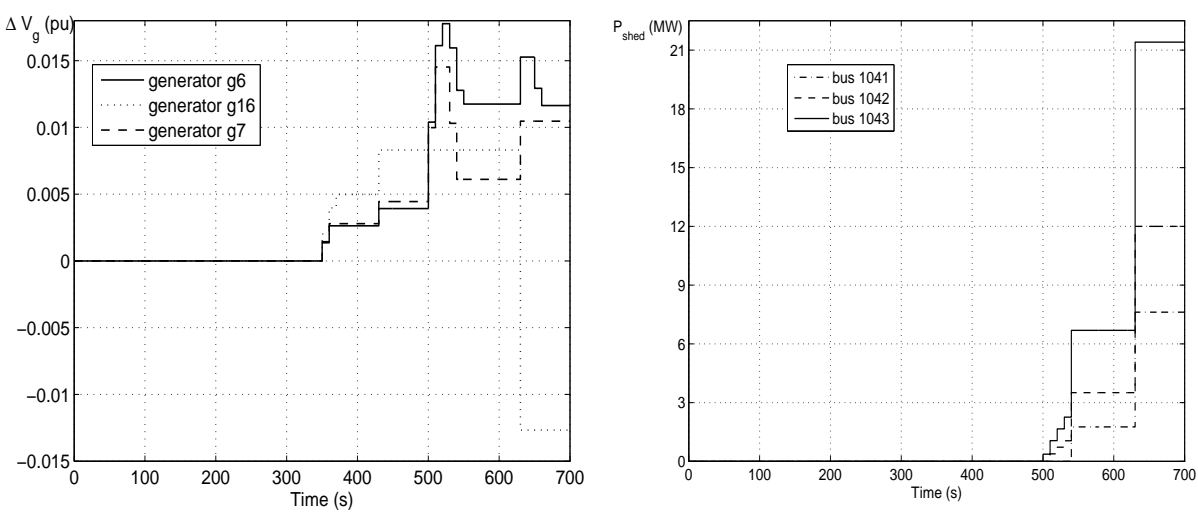

Fig. 10. Case 4: adjustments of some of the generator Fig. 11. Case 4: cumulated load shedding. voltages. able to stabilize the system and voltage collapse occurs at approximately $t=470 \mathrm{~s}$. In the second simulation, signals to control generator g13 were also blocked, leading to system collapse for the SSO approach at $t=290 \mathrm{~s}$, as shown by dashed curve in the same figure. In the third simulation, failures in both shunt switching and g13 voltage control were considered. Figure 14 shows that the RHMSO approach stabilizes the system. Although the failure is not known by the controller, it compensates by resorting to other available controls including shedding a small amount of load (4.1 MW) at bus 1043 at $t=130 \mathrm{~s}$.

Finally, the effect of optimistic generator reactive power limits and uncertainty in load shedding on the performance of both control approaches was examined. Figure 15 illustrates the voltage magnitude at bus 1044 for these sets of simulations.

In the first set of simulations, optimistic reactive power limits of generators are assumed (ranging from +1 to $+7.5 \%$ of the actual value). Simulation results found that even a small error in these limits considerably impacts the performance of SSO and computed controls are not able to stabilize the system. In the second simulation, uncertainty in load shedding is considered. Here the actual reactive power shed is less than the control signal (constant power factor is not preserved). For buses $1022,1041,1042,1044$, and 1045 only $75 \%$ of the reactive load shed control command is actually curtailed and only $50 \%$ is curtailed at buses 1043 and 2031. With the SSO approach the system collapses after $t=590 \mathrm{~s}$. In the final simulation, the RHMSO approach is applied with both the $7.5 \%$ optimistic reactive power limits and same load shedding errors as above and does stabilize the system.

\section{Design Parameters}

A few design parameters are involved in the proposed method. One parameter to set is the sampling period. We tried different sampling periods, ranging form 3 to 15 seconds, for the system studied in this paper. A period of 10 seconds was found acceptable, and it is likely to be in general for practical power systems. It matches the value used in the coordinated secondary voltage control in operation in France [7]. It gives time to telecommunication and for solving the optimization problem with a security margin.
A second parameter to set is the control horizon $K$. Larger values of $K$ lead to smoother control but higher settling times and increased computational effort (there are more eqs. $4 \mathrm{~b}$, $4 \mathrm{c}, 4 \mathrm{~d}, 4 \mathrm{~g}$ to treat). Furthermore, the control sequence should be fast ( $K$ low) enough to be able to counteract voltage instability [4], [5]. On the other hand, small values of $K$ makes the control scheme come closer to the SSO criticized in Section III.

Values of $K$ from 2 to 5 were tried and the best performances were achieved using $K=3$. Figure 16 illustrates the impact of varying $K$ by showing the magnitude of the voltage at bus 1044 . As can be seen, a higher value of $K$ leads to slower system response and significantly longer settling time. This is the consequence of less aggressive control due to slower load power recovery. The system response with different values for the prediction horizon $K^{\prime}$ and the control horizon $K$ is also shown in the figure. It clearly supports the claim in Section IV.A that there is no advantage in choosing $K^{\prime}>K$.

The response of the RHMSO approach was found little sensitive to small variations in the value of $K$, however more significant changes can result in poor performance. For each particular system considered, planning studies would need to be performed to determine an appropriate value to use.

Finally, the rate of change of controls may be limited by specifying constraints of the type (Eq. 4d). The objective is to protect equipment against unacceptable variations. It also acts as a safeguard against an abnormal future system evolution that could hypothetically result from the absence of bounds on $\mathbf{V}^{k+1}, \ldots, \mathbf{V}^{k+K-1}$. The components of $\boldsymbol{\Delta}$ must not be set too low, as it could make the optimization problem (4) infeasible. In our tests the only constraints imposed (on generator voltages) were not active.

\section{J. Computational Burden}

The simulations required solution times of 0.71 seconds for the RHMSO approach and 0.47 for the SSO approach. These are the times elapsed from the call of GAMS in MATLAB until the results of the optimization are returned back from GAMS to MATLAB. For the Nordic32 test system the 


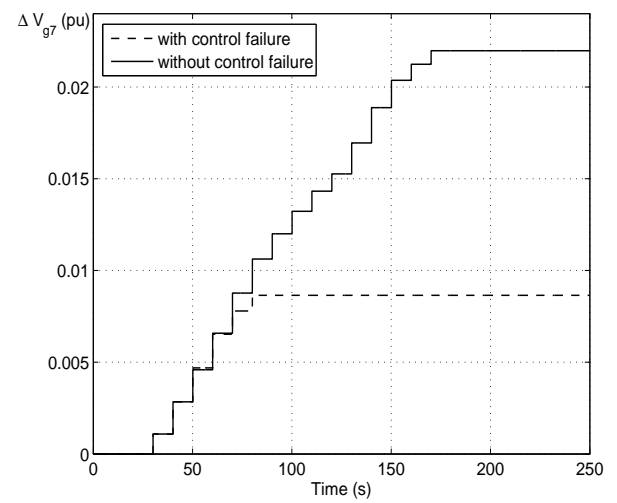

Fig. 13. Case 5: generator g7 control with and without component failure.

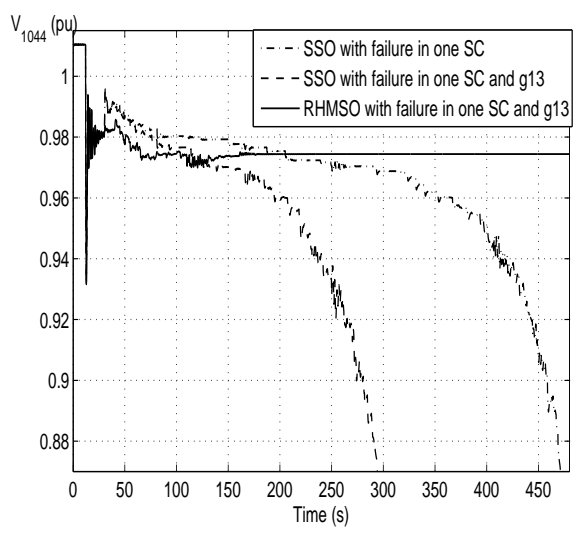

Fig. 14. The voltage response with failures in some controls; SC stands for Shunt Capacitor.

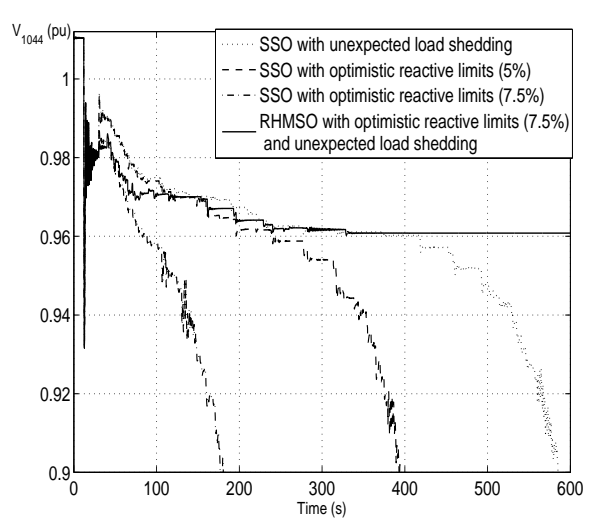

Fig. 15. The voltage response with uncertainties in reactive power and load shedding.

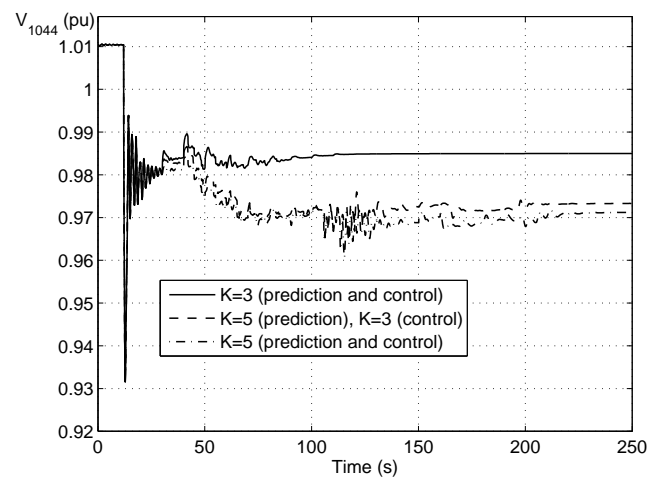

Fig. 16. Illustration on the impact of value of $\mathrm{K}$.

RHMSO approach involves 309 equality constraints, up to 332 inequality constraints, 309 state variables, and up to 30 control variables. For this test system, the SSO approach involves 103 equality constraints, up to 147 inequality constraints, 103 state variables, and up to 30 controls. The optimization problems to be solved for both approaches are relatively small.

The average execution times, as reported by GAMS to solve optimization problem (without time for data transfer) are: 0.027 second for the RHMSO and 0.014 for SSO approach. Using a simple warm-start strategy [29] in multistep optimization considerably decreases execution time to 0.016 second which is only 2 milisecond higher than the execution time in SSO. This strategy consists of initializing the control vectors $\mathbf{u}^{k+1}, \mathbf{u}^{k+2}, \ldots, \mathbf{u}^{k+K-1}$ to the values computed at the previous time step $k-1$, and $\mathbf{u}^{k+K}$ to $\mathbf{u}^{k+K-1}$ in the absence of a better initial guess for this newly introduced unknown. Since the sampling period is 10 seconds, observed IPOPT performances for practical problem are clearly compatible with real-time control requirements.

In a real-life application, in order to decrease the computational complexity, the optimization problem can be solved concentrating on areas close to the disturbance with a simple representation used for other areas. If a utility were to implement the RHMSO approach, the algorithms developed in MATLAB would be replaced with efficient software designed for real-time operation. Such software would include full exploitation of the sparsity found in the problem further reducing the computation time.

\section{CONCLUSION}

To control non viable or unstable transmission voltages, this paper has proposed an algorithm relying on a model typical of optimal power flow but implemented in multiple steps and with a receding horizon, as considered typically in MPC. The test were performed on a detailed dynamic system model in order to consider realistic conditions. It was effective using capacitor switching, generator voltages, and load shedding. The proposed load recovery model used in RHMSO was successful achieving an acceptable control response to stabilize the system. Disturbances associated with line outages and incremental load increases were considered. In both cases, the RHMSO control approach provided stabilization. In situations where a disturbance resulted in low voltage magnitudes, but the system remained stable, the proposed approach did enhance the voltage profile. The robustness of the controller was considered by not applying some control actions. From this simulation, the RHMSO algorithm emphasized control settings which were not subject to this failure, resulting in system stabilization. The RHMSO was compared with the SSO approach and was shown to be more reliable when control failure and uncertainty in reactive limits and load shedding was considered.

\section{REFERENCES}

[1] "Final report on the august 14, 2003 blackout in the united states and canada: Causes and recommendations," US-Canada Power System Outage Task Force, 2004, accessed in June 2010. [Online]. Available: https://reports.energy.gov/

[2] "Final report: System disturbance on 4 november, 2006," Union for the Co-ordination of Transmission of Electricity, 2007, accessed in June 2010. [Online]. Available: http://www.entsoe.eu/fileadmin/user_upload/ _library/publications/ce/otherreports

[3] $\bar{C}$. W. Taylor, Power system voltage stability. New York: McGraw-Hill, 1994.

[4] T. Van Cutsem and C. Vournas, Voltage Stability of Electric Power Systems. Boston: Kluwer, 1998.

[5] — , "Emergency voltage stability controls: an overview," 2007 IEEE Power Engineering Society General Meeting, 2007. 
[6] S.-K. Chang, F. Albuyeh, M. Gilles, G. Marks, and K. Kato, "Optimal real-time voltage control," IEEE Trans. Power Syst., vol. 5, no. 3, pp. $750-8$, Aug. 1990.

[7] H. Vu, P. Pruvot, C. Launay, and Y. Harmand, "An improved voltage control on large-scale power system," IEEE Trans. Power Syst., vol. 11, no. 3, pp. 1295 - 303, 1996.

[8] D. Popovic, V. Levi, and Z. Gorecan, "Co-ordination of emergency secondary-voltage control and load shedding to prevent voltage instability," IEE Proceedings-Generation, Transmission and Distribution, vol. 144, no. 3, pp. 293 - 300, 1997.

[9] B. Marinescu and H. Bourles, "Robust predictive control for the flexible coordinated secondary voltage control of large-scale power systems," IEEE Trans. Power Syst., vol. 14, no. 4, pp. 1262 - 8, 1999.

[10] M. Zima and G. Andersson, "Stability assessment and emergency control method using trajectory sensitivities," 2003 IEEE Bologna PowerTech, vol. 2, 2003.

[11] M. Larsson and D. Karlsson, "Coordinated system protection scheme against voltage collapse using heuristic search and predictive control," IEEE Trans. Power Syst., vol. 18, no. 3, pp. 1001 - 6, 2003.

[12] J. Wen, Q. Wu, D. Turner, S. Cheng, and J. Fitch, "Optimal coordinated voltage control for power system voltage stability," IEEE Trans. Power Syst., vol. 19, no. 2, pp. 1115 - 22, 2004.

[13] I. Hiskens and B. Gong, "Voltage stability enhancement via model predictive control of load," Bulk Power System Dynamics and Control VI, pp. 117 - 124, 2004.

[14] A. Beccuti, T. Demiray, G. Andersson, and M. Morari, "A lagrangian decomposition algorithm for optimal emergency voltage control," IEEE Trans. Power Syst., vol. 25, no. 4, pp. 1769 - 1779, 2010.

[15] L. Jin, R. Kumar, and N. Elia, "Model predictive control-based real-time power system protection schemes," IEEE Trans. Power Syst., vol. 25, no. 2, pp. $988-998,2010$.

[16] J. M. Maciejowski, Predictive Control with Constraints. Prentice Hall, 2002.

[17] M. Glavic and T. Van Cutsem, "Wide-area detection of voltage instability from synchronized phasor measurements. part I: Principle," IEEE Trans. Power Syst., vol. 24, no. 3, pp. 1408 - 1416, 2009.

[18] D. Karlsson and D. Hill, "Modelling and identification of nonlinear dynamic loads in power systems," IEEE Trans. Power Syst., vol. 9, no. 1, pp. 157 - 66, 1994.

[19] W. Xu and Y. Mansour, "Voltage stability analysis using generic dynamic load models," IEEE Trans. Power Syst., vol. 9, no. 1, pp. 479 - 93, 1994.

[20] S. Joe, Qin and T. A. Badgwell, "An overview of industrial model predictive control technology," Chemical Process Control, vol. 93, no. 316, pp. $232-256,1997$.

[21] D. Mayne, J. Rawlings, C. Rao, and P. Scokaert, "Constrained model predictive control: stability and optimality," Automatica, vol. 36, no. 6, pp. $789-814,2000$.

[22] M. Stubbe (Convener), "Long-term dynamics phase II," CIGRE Task Force, Tech. Rep., 1995.

[23] M. Glavic and T. Van Cutsem, "Wide-area detection of voltage instability from synchronized phasor measurements. part II: Simulation results," IEEE Trans. Power Syst., vol. 24, no. 3, pp. 1417 - 1425, 2009.

[24] C. Vournas, E. Potamianakis, C. Moors, and T. Van Cutsem, "An educational simulation tool for power system control and stability," IEEE Trans. Power Syst., vol. 19, no. 1, pp. 48 - 55, 2004.

[25] "General algebraic modeling system." [Online]. Available: http: //www.gams.com

[26] M. C. Ferris, "Matlab and GAMS: Interfacing optimization and vizualization software," 2005. [Online]. Available: http://www.cs.wisc. edu/math-prog/matlab.html

[27] A. Wachter and L. T. Biegler, "On the implementation of an interiorpoint filter line-search algorithm for large-scale nonlinear programming," Mathematical Programming, vol. 106, no. 1, pp. 25 - 57, 2006.

[28] L. Biegler and V. Zavala, "Large-scale nonlinear programming using ipopt: an integrating framework for enterprise-wide dynamic optimization," Computers \& Chemical Engineering, vol. 33, no. 3, pp. 575 - 82, 2009.

[29] M. N. Zeilinger, C. N. Jones, D. M. Raimondo, and M. Morari, "Realtime mpc - stability through robust mpc design," 48th IEEE Conference on Decision and Control, pp. 3980 - 3986, 2009.

Mevludin Glavic (M'04, SM'07) received the M.Sc. and Ph.D. degrees from the Univ. of Belgrade (Serbia) and Tuzla (Bosnia) in 1991 and 1997, respectively. He was with the Univ. of Wisconsin-Madison (1999-2000, as a Fulbright postdoctoral scholar) and with the Univ. of Liège (2001-2004 as research fellow, 2006-2009 as visiting professor). Since 2005 he has been working as consultant for Deling-doo (Bosnia) in projects with AREVA T\&D
(France), Suez Tractebel Eng. (Belgium), and Quanta Technology (USA). His research interests are in power system dynamics, stability and control.

Mahdi Hajian (M'07) received the B.Sc. and M.Sc. degrees from the Isfahan University of Technology (2005) and the Sharif University of Technology (2007), Iran. Currently, he is a Ph.D. student at the Department of Electrical and Computer Engineering, University of Calgary. His research interests are in optimization and control of power systems.

William (Bill) Rosehart (M'01, SM'06) received his B.Sc., M.Sc. and Ph.D. degrees in Electrical Engineering from the University of Waterloo (1996, 1997, 2001). He has been with the Schulich School of Engineering at the University of Calgary since 2001, where he is currently a Professor in the Department of Electrical and Computer Engineering. His research interests are in optimization and stability analysis of energy systems.

Thierry Van Cutsem (F'05) graduated in Electrical-Mechanical Engineering from the Univ. of Liège (Belgium), where he obtained the Ph.D. degree and he is now adjunct professor. Since 1980, he has been with the Fund for Scientific Research (FNRS), of which he is now a Research Director. His research interests are in power system dynamics, stability, security, simulation and optimization, in particular voltage stability and security. 